was non-invasively assessed by Pulse pen device and Alx was evaluated by tonometry. Statistical analysis was done with SPSS v22 software, we calculated mean and standard deviation, for continuous variables we used Student $t$ test, categorical variables were analyzed by using chi-square or Fisher's exact test. The correlation of AS and clinical variables was assessed with Spearman's correlation.

Results: Forty seven patients were included and compared with 39 healthy subjects; mean age of study group was $48 \pm 14$. vs control group $47 \pm 13.7 \quad(p=0.08)$ $93 \%$ were female. Prevalence of AS was $11 \%$ vs $3 \% p=0.039$. AS was more frequent in limited systemic sclerosis sub-group and we found correlation with abnormal capillaroscopy, Rho $0.292 \mathrm{p}=0.04$.

Conclusions: Arterial stiffness is more prevalent in patients with limited systemic sclerosis and association with abnormal caillaroscopy suggest that both macro and microvascular damage is present in these patients and could explain the presence of early atherosclerosis and increased risk of cardiovascular disease.

References:

[1] Vargas John, Lafyatis Robert. Etiology and pathogenesis of systemic sclerosis. Marc Hochberg. Texbook of Rheumatology (Sixth Edit). Elsevier, 2015. 11771245.

[2] Ngian G.-S., Sahhar J., Wicks I., Van Doornum, S. Arterial stiffness is increased in systemic sclerosis: A cross-sectional comparison with matched controls. Arthritis and Rheumatism 2012. 64, S301.

[3] Colaci M., Giuggioli D., Manfredi A., Sebastiani M. Aortic pulse wave velocity measurement in systemic sclerosis patients, Rheumatism 2012. 64(6), 360367.

[4] Man A., Zhu Y., Zhang Y., Dubreuil M., Rho Y. H., Peloquin C. Choi, H. K. The risk of cardiovascular disease in systemic sclerosis: a population-based cohort study. Annals of the Rheumatic Diseases 2013. 72(7), 1188-93.

Disclosure of Interest: None declared

DOI: 10.1136/annrheumdis-2017-eular.3345

\section{AB0642 THICKNESS OF THE INTIMA-MEDIA COMPLEX AND DOPPLER VELOCITY PARAMETERS IN SCLERODERMA PATIENTS}

K. Rutka ${ }^{1}$, E. Gindzienska-Sieskiewicz ${ }^{2}$, R. Milewski ${ }^{3}$, S. Sierakowski ${ }^{2}$ U. Lebkowska ${ }^{1} .{ }^{1}$ Radiology; ${ }^{2}$ Rheumatology; ${ }^{3}$ Statistics and Medical Informatics, Medical University of Bialystok, Bialystok, Poland

Background: Systemic sclerosis (Ssc) is a chronic inflammatory autoimmune disease, that involves various tissues and organs, including the cardiovascular system. Ssc patients might suffer from a number of complications - including cardiovascular system diseases. Diagnostic imaging is an important tool in assessment of vascular lesions, and ultrasound (including Doppler ultrasound) is one of the most important examinations, which allow to scan for presence of atherotic lesions, assess the intima-media complex thickness as well as provide measurements of the blood flow parameters.

Objectives: The aim of study was to determine if there is any difference in the intima-media complex thickness as well as in blood flow parameters measured using Doppler ultrasound examination in scleroderma patients and the general population.

Methods: 35 patients, aged 19-75, with diagnosed systemic scleroderma were examined using a Doppler ultrasound examination. Thickness of intima-media complex (IMT) approximately 2 centimeters from the carotid bulb was assessed for both right and left common carotid artery (CCA). The standard parameters of blood flow were measured - including peak systolic velocity (PSV), end diastolic velocity (EDV), as well as resistive index (RI), pulsative index (PI) and standard deviation (SD) was measured in the common carotid arteries, internal carotid arteries (ICA) as well as in the vertebral arteries (VA).

Results: The mean IMT value in CCA was approximately $0.63 \mathrm{~mm}(0.35-$ $0.9 \mathrm{~mm})$.

The mean, minimal and maximal values measured in CCA equalled respectively: PSV - $68.7 \mathrm{~cm} / \mathrm{s}(22.4-94 \mathrm{~cm} / \mathrm{s})$, EDV - 19,7 cm/s $(4.7-30.35 \mathrm{~cm} / \mathrm{s}), \mathrm{PI}-$ $1.95(0.41-2,09)$ and $\mathrm{RI}-0.68(0.28-0.81)$. In ICA the measured values were as follows: PSV $-68.4 \mathrm{~cm} / \mathrm{s}(38.65-96.9 \mathrm{~cm} / \mathrm{s})$; EDV $-23.6 \mathrm{~cm} / \mathrm{s}(9.05-38.6$ $\mathrm{cm} / \mathrm{s}), \mathrm{PI}-1.16(0.76-1.71)$ and $\mathrm{RI}-0.65(0.37-0.79)$; in VA PSV $-46.5 \mathrm{~cm} / \mathrm{s}$ $(26.75-75.35 \mathrm{~cm} / \mathrm{s}) ; \mathrm{EDV}-12.4 \mathrm{~cm} / \mathrm{s}(6.1-22 \mathrm{~cm} / \mathrm{s}), \mathrm{PI}-1.38(0.6-2.28)$ and $\mathrm{RI}$ $-0.73(0.34-1.33)$.

A positive correlation between the age of examined subjects and the IMT was found $(p=0.002 ; R=0.49$ ). Additionaly, a negative correlation between the IMT and the EDV was found $(p=0.008, R=-0.44)$.

Conclusions: The mean thickness of intima-media complex in the examined group of SSc patients is within the values that were established for a healthy population, however a further investigation, including a control group study will allow to evaluate whether there is no correlation between systemic sclerosis and increased IMT.

A negative correlation between IMT and EDV was shown, which is an interesting finding, and could be confirmed in a control group study and on a larger group of patiens.

References:

[1] Kochanowicz J. et al Normal reference values of ratios of blood flow velocities in internal carotid artery to those in common carotid artery using Doppler sonography. J Clin Ultrasound. 2009 May;37(4):208-11.

[2] Buljan K et al. Relationship between Age and Thickness of Carotid Arteries in a Population without Risk Factors for Atherosclerosis. Coll Antropol. 2015 Sep;39(3):779-84.

[3] Yazici B. et al. Cerebral blood flow measurements of the extracranial carotid and vertebral arteries with Doppler ultrasonography in healthy adults. Diagn Interv Radiol. 2005 Dec;11(4):195-8.

Disclosure of Interest: None declared

DOI: 10.1136/annrheumdis-2017-eular.6296

\section{AB0643 MALIGNANCY SCREENING IN AUTOIMMUNE MYOSITIS AMONGST AUSTRALIAN RHEUMATOLOGISTS}

K. Dutton ${ }^{1}$, M. Soden ${ }^{2} .{ }^{1}$ Rheumatology, RBWH, Brisbane; ${ }^{2}$ Rheumatology, Townsville Hospital, Douglas, Australia

Background: The association between cancer and autoimmune myositis is well established and has lead to the common practice of malignancy screening in asymptomatic individuals. The international literature advocates widely for cancer screening in autoimmune myositis however no consensus or quideline has been published to set forth a process for screening standardisation (1). Malignancy screening is a complicated topic and recommendations in favour of screening should in principle be based on a judicious assessment of the evidence in terms of the benefits, risks and costs. In inflammatory myositis there is currently insufficient evidence to support any recommendation with respect to cancer screening. In the absence of clinical guideline and quality evidence, our study aimed to establish the current trends in malignancy screening amongst Australian Rheumatologists. Objectives: To explore the current trends in malignancy screening in autoimmune myositis amongst Australian Rheumatologists using an online questionnaire. Methods: Research approval was granted by The Townsville Hospital. An invitation email containing the survey weblink was sent twice to 386 Australian Rheumatologists between August 2015 and August 2016. Voluntary participation and anonymity were guaranteed. The questionnaire contained a fixed set of multiple choice questions that requested data on respondent demographics, practice setting and screening preference, practice and concerns. Open entry comment was an option throughout the questionnaire. Fifty-eight Rheumatologists, 1 Immunologist and 1 Paediatric Rheumatologist responded (16\% response rate). There were 3 survey dropouts. The data was pooled, coded and analysed using statistical software. All data was included in the analysis.

Results: Most respondents $(\mathrm{N}=58)$ were in private $(67 \%)$ and/or public practice $(68 \%)$, in practice for $>10$ years $(70 \%)$, conducted cancer screening $(93 \%)$ and were "very" or "somewhat" confident in their screening practice $(90 \%)$. The majority $(72 \%)$ performed cancer screening independent of patient characteristics. Determinants that triggered screening (in descending order of popularity) were: tobacco use $(\mathrm{N}=11)$, history of cancer $(\mathrm{N}=10)$, age $>40(\mathrm{~N}=7)$, cancer family history $(\mathrm{N}=7)$, age $>50(\mathrm{~N}=3)$ and age $>60(\mathrm{~N}=1)$. Most respondents indicated preference to order screening tests (in descending order of popularity): mammogram $(81 \%)$; CT chest \& abdomen (78\%); myeloma screen $(70 \%)$; chest x-ray $(69 \%)$, serum PSA $(67 \%)$, PAP smear $(54 \%)$, colonoscope $(44 \%)$, LDH (41\%), pelvic USS $(33 \%)$, gastroscope $(33 \%)$, FOBT $(33 \%)$, tumour markers $(28 \%)$, CT neck $(17 \%)$, nuclear bone scan $(15 \%)$, PET CT $(4 \%)$ \& testicular USS $(2 \%)$. Respondents $(\mathrm{N}=57)$ indicated that cancer screening was problematic due to a lack of clinical practice consensus \& guideline $(77 \%)$, test selection knowledge $(37 \%)$ and knowledge regarding repeated screening (53\%). The potential for harm in conducting screening was identified to be a problem by most respondents $(62 \%)$. Conclusions: The practice of malignancy screening in autoimmune myositis amongst Australian Rheumatologists is highly variable. Practice is driven by patient factors and clinician preferences. The cancer screening process is felt on several fronts to have inherent problems. Guideline, consensus and further research is needed in this area to address the challenges and evidence gap.

References:

[1] Masiak, A et al. (2016). Clinical characteristics of patients with anti-TIF1-y Abs. Reumatologia. 54(1): 14-18.

Disclosure of Interest: None declared

DOI: 10.1136/annrheumdis-2017-eular.1148

\section{AB0644 FINGERPRINT ABNORMALITIES IN SYSTEMIC SCLEROSIS: A SINGLE CENTER SURVEY FROM INDIA}

K. Chanakya, P.K. Devarasetti, R.V.P. Irlapati, L. Rajasekhar. Rheumatology, Nizams institute of medical sciences, Hyderabad, India

Background: Fingerprint [FP] abnormalities are known in patients with Systemic Sclerosis [SSc]. Little has been described about their frequency, systemic associations and social impact in literature.

Objectives: To study the fingerprint abnormalities in Systemic Sclerosis patients Methods: Raynaud's phenomenon [RP] was taken as the inclusion criteria. Patients with SSc [limited LcSSc and diffuse DcSSc], SSc overlap with other Connective Tissue Diseases [CTDs] and other CTDs with RP were screened for FP abnormalities using a Standardization Testing and Quality Certification (STQC) Directorate certified biometric FP scanner. FP quality assessment was done by recording The National Institute of Standards and Technology [NIST] finger print image quality [NFIQ] scores ${ }^{1}$. NFIQ's 5 levels of quality are intended to be predictive of fingerprint matching. $\mathrm{NFIQ}=1$ indicates high quality samples and $\mathrm{NFIQ}=5$ indicates poor quality samples. Other associated systemic features of 
the disease were noted. Also the social difficulties due to fingerprint abnormalities were noted. Healthy controls with no RP were included for comparison.

Results: 40 consecutive patients with RP attending Rheumatology outpatient services of our institute were screened for FP abnormalities. 29 with SSc [20DcSSc, 9-LcSSc], 8 with overlap syndromes and 1 each of SLE, Undifferentiated Vasculitis and Undifferentiated CTD. It was noted prior to screening that 19 patients experienced some difficulty in the past with biometric recognition of their FPs at various times. On screening with biometric scanner, 15 of 40 [37.5\%] had FP abnormalities in the form of non recognition of at least one finger with a median of 2 [range 1-6 fingers]. Of these 15, seven had DcSSc, six had LcSSc and two with overlap syndromes. The mean NFIQ score of these 15 patients was 4.5 [poor] and the mean NFIQ scores in SSc was 3.8. Eleven [27.5\%] patients could not get government Identity cards based of FP scanning, four could not avail various government benefit schemes which needed their fingerprints as identity. Sixteen [40\%] had history of digital vasculopathy in the form of digital pits, digital ischemia or ulcers. PAH was found in one and eight had Interstitial lung disease. Among the 10 controls all FPs were recognized and the mean NFIQ score was 2.2 indicating a better quality of FPs.

Conclusions: Fingerprint abnormalities occur frequently in patients with systemic sclerosis causing social disabilities in few. The quality of FPs in SSc patients is poor. Raynaud's phenomenon and vasculopathy are frequently associated. Documentation of this abnormality should allow the use of other biometric tools for personal identification.

\section{References:}

[1] E. Tabassi, C. Wilson, C. Watson, "Fingerprint Image Quality", NIST Internal Report 7151, National Institute for Standards and Technology, 2004.

Disclosure of Interest: None declared

DOI: 10.1136/annrheumdis-2017-eular.3860

\section{AB0645 LYMPHOCYTE SUBSETS T, B AND NK CELLS IN SYSTEMIC SCLEROSIS}

\section{Schneider $^{1}$, V. $\operatorname{Hax}^{1}$, N. Marcondes ${ }^{1}$, R.M. Xavier ${ }^{2}$, R. Chakr ${ }^{1}$.}

\section{${ }^{1}$ Rheumatology; ${ }^{2}$ Rheumatologi, HCPA, Porto Alegre, Brazil}

Background: Systemic sclerosis (SSc) is a rare multisystem disease with underlying immune mechanisms, whose pathogenesis remains unclear. Few previous reports have evaluated lymphocyte subpopulations in SSc and your results are conflicting.

Objectives: The present study aimed to analyze the lymphocyte subsets in SSc patients in comparison to healthy individuals.

Methods: Peripheral blood (PB) samples to analyze lymphocyte subsets were obtained from a non-random convenience sample of 20 SSc patients. Twenty healthy individuals recruited from the blood bank were used as sex and agematched controls. Blood samples were analyzed by flow cytometry for total T cells, CD4+ and CD8+ T cells subsets, CD19+ B cells and total NK cells. Statistical analyses were performed using the IBM Statistical Package for Social Sciences (SPSS 18.0). Data are expressed as mean \pm SD and median and range. Non-parametric Mann-Whitney $U$ test was used for analyses of the flow cytometry. A probability $p<0.05$ was considered statistically significant.

Results: The mean (SD) age of SSc patients was 57.9 (14.2) years, 95\% were female and $31.6 \%$ presented diffuse cutaneous SSc (dcSSc). Patients presented a lower mean total lymphocyte count compared to healthy controls $(23.7 \%$ vs. $29.6 \%, p=0.026$ ) (Table 1.). No statistically significant differences were found in the percentages or the absolute numbers of T, B or NK cells.

\begin{tabular}{lcccc}
\hline & & Patients & Controls & $\mathrm{p}^{\mathrm{a}}$ \\
\hline Leukocytes & & $8.02 \pm 1.51$ & $6.79 \pm 3.39$ & 0.108 \\
Lymphocytes & $\%$ & $23.53 \pm 14.06$ & $29.97 \pm 15.08$ & $0.026^{*}$ \\
& Absolute & $1.70 \pm 1.59$ & $2.06 \pm 0.03$ & 0.512 \\
CD45 & $\%$ & $99.79 \pm 0.11$ & $99.71 \pm 0.11$ & 0.165 \\
CD3 & $\%$ & $71.20 \pm 3.57$ & $75.11 \pm 1.79$ & 0.398 \\
& Absolute & $1.35 \pm 1.20$ & $1.60 \pm 0.07$ & 0.478 \\
CD4 & $\%$ & $48.59 \pm 4.09$ & $47.76 \pm 8.78$ & 0.289 \\
& Absolute & $0.85 \pm 0.94$ & $0.99 \pm 0.12$ & 0.620 \\
CD8 & $\%$ & $25.10 \pm 0.04$ & $28.38 \pm 1.19$ & 0.277 \\
& Absolute & $0.48 \pm 0.29$ & $0.51 \pm 0.03$ & 0.301 \\
CD19 & $\%$ & $8.89 \pm 11.57$ & $10.44 \pm 2.07$ & 0.478 \\
& Absolute & $0.20 \pm 0.02$ & $0.17 \pm 0.03$ & 0.512 \\
NK & $\%$ & $6.73 \pm 8.39$ & $6.71 \pm 5.40$ & 0.883 \\
& Absolute & $0.14 \pm 0.35$ & $0.14 \pm 0.09$ & 0.947 \\
Ratio & T/B & $8.54 \pm 4.11$ & $7.31 \pm 2.3$ & 0.383 \\
& CD4/CD8 & $1.93 \pm 0.23$ & $1.73 \pm 0.33$ & 0.157 \\
\hline
\end{tabular}

Conclusions: Our data support previous reports indicating that depletion of lymphocyte in the PB of SSc patients. However, we found no significant difference in relation to lymphocyte subtypes, which differs from the literature data.

\section{References:}

[1] T and NK Cell Phenotypic Abnormalities in Systemic Sclerosis: a Cohort Study and a Comprehensive Literature Review. Almeida et al, 2015.

[2] Liu M, Wu W, Sun X, Yang J, Xu J, Fu W, Li M. New insights into CD4+ T cell abnormalities in systemic sclerosis. Cytokine \& Growth Factor Reviews, 2016; 28:31-36.

[3] Gambichler T, Tigges C, Burkert B, Höxtermann S, Altmeyer P, Kreuter A.
Absolute count of $T$ and $B$ lymphocyte subsets is decreased in systemic sclerosis. Eur J Med Res 2010; 15:44-46.

Disclosure of Interest: None declared

DOI: 10.1136/annrheumdis-2017-eular.7032

\section{AB0646 DETERMINANTS OF QUALITY OF LIFE IN SYSTEMIC} SCLEROSIS AND PATIENT'S PERCEPTION OF THEIR ILLNESS

L. Groseanu $^{1,2}$, A. Balanescu ${ }^{1,2}$, D. Predeteanu ${ }^{1,2}$, D. Opris-Belinski ${ }^{1,2}$,

V. Bojinca ${ }^{1,2}$, I. Saulescu ${ }^{1,2}$, A. Borangiu ${ }^{1,2}$, D. Mazilu ${ }^{1,2}$, C. Constantinescu ${ }^{1,2}$,

F. Berghea ${ }^{1,2}, \mathrm{R}$. Ionescu ${ }^{1,2}$. ${ }^{1}$ Internal Medicine and Rheumatology, Sf Maria Clinical Hospital; ${ }^{2}$ Department 5- Internal Medicine, University of Medicine and Pharmacy Carol Davila, Bucharest, Romania

Background: Systemic sclerosis (SSc) is a chronic multi-system autoimmune disease associated with disability and reduced quality of life.

Objectives: The purpose of this study was to assess health-related quality of life and disease perception in a group of SSc patients.

Methods: We performed a case-control study on 50 SSc patients from EUSTAR cohort 096. Socio-demographic data, disease characteristics and self-assessment questionnaires: Health assesment questionnaire (HAQ), EuroQol-5D (EQ5D) and the Brief Illness Perception Questionnaire were collected.

Results: The group included 41 females, 31 limited SSc subsets.

Medium HAQ value was $0.9(0.6)$. Patients with higher Rodnan score $(p=0.002)$, synovitis $(p=0.02)$, late capillaroscopic pattern $(p=0.02)$, muscle weakness $(p=0.001)$, gastrointestinal involvement $(p=0.01)$ and those on immunosuppressants $(p=0.02)$ have a poor life quality.

According to EQ-5D, the quality of life was related to specific organ involvement. $48 \%$ of the patients had some mobility problems, $6 \%$ were confined to bed; mobility was influenced by lung involvement $(p=0.008)$, digital ulcers $(p=0.03)$ and Medsger score $(p=0.01)$. $48 \%$ of the patients had some self-care problems and $8 \%$ were not able to wash/dry themselves; self-care was influenced by the Rodnan score $(p=0.02)$, diffuse subset $(p=0.02)$, muscle weakness $(p=0.03)$ and gastrointestinal involvement $(p=0.021)$. $64 \%$ of the patients had some problems in performing usual activities and $16 \%$ were not able to perform them; the performance was influenced by disease subset $(p=0.01)$, Medsger score $(p=0.02)$, cardiac involvement $(p=0.02)$ and the use of immunosuppressants $(p=0.01) .52 \%$ of the patients had some and $38 \%$ had extreme pain/disconfort; $66 \%$ of the patients were moderately and $20 \%$ were extremly anxious/depressed. Both were related to digital ulcers $(p=0.01$, respectively $p=0.045)$

The illness had a great impact on patients life $7.3(2.5) / 10$. The main determinant was pulmonary fibrosis $(p=0.04)$. The patients consider that their disease will continue for quite a long time $8.7(2.6) / 10$. Most of the patiens do not feel to have a good control on their disease 6.3 (3.3)/10 and unfortunately they do not think that the treatment is very helpful $7.9(2.7) / 10$. The intensity of the symptoms is quite severe $7.5(2.7) / 10$, related to digital ulcers $(p=0.04)$ and gastrointestinal involvement $(\mathrm{p}=0.02)$. Patients are very concerned about their disease 9.1 (2.3)/10, most of them being emotionally affected 7.6 (2.6).

Conclusions: This study confirms the presence and magnitude of impaired life quality in patients with SSc with impact on mobility, self-care, usual activities. The major determinants were the extend of skin involvement, musculoarticular, gastrointestinal involvement and digital ulcers. Often patients are anxious/depressed, had a high pain intensity and the perception of this illness is pessimistic.

References:

[1] Hudson M, Canadian Scleroderma Research Group Health-related quality of life in systemic sclerosis: a systematic review. Arthritis Rheum. 2009;61(8):1112-2

[2] Frantz $\mathrm{C}$ et al. Impaired quality of life in systemic sclerosis and patient perception of the disease: A large international survey. Semin Arthritis Rheum. 2016;46(1):115-23.

Disclosure of Interest: None declared

DOI: 10.1136/annrheumdis-2017-eular.4178

\section{AB0647 DIAGNOSIS OF SYSTEMIC SCLEROSIS - "A TANGLED STORY"}

L. Groseanu $^{1,2}$, A. Dima ${ }^{1}$, T. Gudu ${ }^{1}$, A. Balanescu ${ }^{1,2}$, D. Predeteanu ${ }^{1,2}$, D. Opris-Belinski ${ }^{1,2}$, I. Saulescu ${ }^{1,2}$, A. Borangiu ${ }^{1,2}$, C. Constantinescu ${ }^{1,2}$, D. Mazilu ${ }^{1,2}$, M.M. Negru ${ }^{1,2}$, F. Berghea ${ }^{1,2}$, V. Vlad ${ }^{1}$, C. Cobilinschi ${ }^{2}$, M. Abobului ${ }^{1,2}$, R. Ionescu ${ }^{1,2} .{ }^{1}$ Internal Medicine and Rheumatology, Sf Maria Clinical Hospital; ${ }^{2}$ Department 5 - Internal Medicine, University of Medicine and Pharmacy Carol Davila, Bucharest, Romania

Background: Proper diagnosis of scleroderma is often long and difficult, since it is such a rare disease, and one which few doctors or patients are familiar with. Objectives: To establish the interval between the symptoms' onset of systemic sclerosis (SSc) and what type of investigations are performed until the patients reach the final diagnosis of a rheumatologist

Methods: This is a cross-sectional study that included randomly selected patients with a diagnosis of SSc which were evaluated based on a questionnaire about symptoms at onset, specific consults and investigations. Descriptive statistics were used.

Results: The study group included 47 patients, of which only 5 were males and 17 from rural areas. The medium age was $53(14.4)$ years. 